\title{
Interaction of the pile with the sagging loess soil
}

\author{
Zaven Ter-Martirosyan ${ }^{1, *}$, and Ivan Luzin ${ }^{1}$ \\ ${ }^{1}$ Moscow State University of Civil Engineering, Yaroslavskoe shosse, 26, Moscow, 129337, Russia
}

\begin{abstract}
The article presents the formulation and solution of the problem in the interaction of a pile with a two-layered base, the upper layer of which settles due to the moistening by the filtrational flow (Darcy's law) or moisture transfer (Fick's law), and the lower layer is bearing, possesses relatively strong and deformation properties, which pushes against.

It is shown that due to subsidence of the surrounding soil, negative friction occurs on the lateral surface of the pile, which causes additional draft and force at the level of the heel of the pile. As a calculation, the geomechanical model of the ground cylinder, containing the pile and perceiving the load from the grillage as part of the pile-slab foundation, is considered.
\end{abstract}

\section{Introduction}

It is known that with a limited thickness of the subsidence layer underlain by comparatively dense soils with a strain modulus of at least $40 \mathrm{MPa}$, a pile foundation is often used that cuts through the subsidence layers and is supported by underlying dense layers. In the design of such foundations, the most important tasks are to quantify the load-bearing capacity of piles and their possible subsidence with the total or partial water saturation of the surrounding soil by a downward flow in accordance with Darcy's law or Fick's law, respectively. In this paper, we consider the formulation and solution of the problem of interaction between the pile and the surrounding subsidence ground when the soils are moistened to full or partial water saturation, taking into account the change in deformation and strength properties in accordance with the results of laboratory and field studies. We consider the SSC of a single cell as part of a pile foundation foundation, consisting of a thick-walled ground cylinder that accommodates the pile and receives the load from the grillage.

\section{Precipitation of the subsidence bed in time when it is moistened through the boundary plane}

It is known that in the event of anthropogenic flooding and flooding of loess soils, water is saturated and the process of subsidence develops. It can have disastrous consequences for

\footnotetext{
* Corresponding author: gic-mgsu@mail.ru
} 
the environment, engineering communications and the foundations of the foundations of buildings and structures.

The process of water penetration through the boundary plane has a complex hydrogeomechanical character and requires a quantitative evaluation. The flow of descending water at the water saturation front, $z(t)$, is determined by the change in water content in the elementary layer $d z$ during the time $d t$ and is determined by the expression of the form:

$$
Q(z) \frac{d z}{d t}=k_{f} \frac{d z}{d t}
$$

$Q(z)$ - change of water content in a single layer of soil.

$$
Q(t)=\frac{\gamma(t)}{\gamma_{s}} e_{0}(z)\left(1-S_{r}(z)\right)-\varepsilon_{s e}(z)
$$

where $A_{0}(t)$ is the drawdown coefficient, $m_{s e}$ - the relative compressibility of the moistened soil, $\gamma_{s e}$ - the specific gravity of the soil in the water-saturated state, $e_{0}$ - the initial porosity coefficient, $k_{f}$ - the filtration coefficient.

The precipitation of the layer in accordance with (2) will be equal to

$$
S_{1}^{\max }=l_{1}\left(A_{0}+m_{s e}\left(p+\gamma_{s e} l_{1} / 2\right)\right.
$$

In the case of a homogeneous layer, we get that

$$
Q(t)=\frac{\gamma_{d}}{\gamma_{s}} l_{0}(z)\left(1-S_{r}\right)-A_{0}-m_{s e}\left(p+\gamma_{s e} z\right)
$$

Then (1) will be written as

$$
K=\frac{z+H_{0}+H_{k}}{z}=(m-n z) \frac{d z}{d t}
$$

where $H_{k}$ is the vacuum head at the soaking front, which depends on the initial soil moisture, and

$$
m=\frac{\gamma_{d}}{\gamma_{s}} l_{0}(1-S r)-A_{0}-m_{s e} p ; n=m_{s e} \gamma_{s e} ; \gamma_{s e}=\gamma_{d}\left(1+W_{n}\right)
$$

The solution of (2.1) with allowance for the initial condition $\mathrm{z}(0)=0$ has the form:

$$
\frac{z}{H^{*}}-\ln \left(1+\frac{z}{H^{*}}\right)-\frac{n}{m}\left[\frac{z}{2 H^{*}}\left(z-2 H^{*}\right)+H^{*} \ln \left(1+\frac{z}{H^{*}}\right)\right]=\frac{k_{f} t}{m H^{*}},
$$

where $H^{*}=H_{0}+H_{k}$.

In the absence of drawdown, i.e. for $n=0$, this solution coincides with the solution obtained by P.Ya. Semiboyarinova-Cochina [4], in the form: 


$$
\frac{z}{H *}-\ln \left(1+\frac{z}{H^{*}}\right)=\frac{k_{f} t}{m H^{*}} .
$$

From the solution obtained by us it is seen that $z(t)$ grows with a damped velocity in time (Figure 2). Knowing $z(t)$, it is easy to determine the amount of subsidence of the saturated layer $z(t)$, and also the drawdown at $z=\left(t^{*}\right)=l_{1}$

$$
S_{s l}(t)=z(t) \cdot \varepsilon_{s l}=z(t) \cdot\left[A_{0}+m_{s t}\left(p+\gamma_{s l} \cdot z(y)\right)\right]
$$

where $A_{0}$ - coefficient of subsidence.

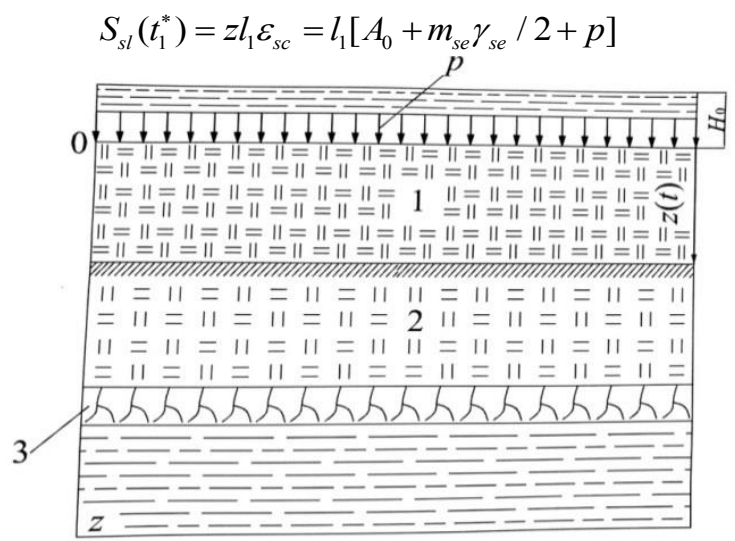

Fig. 1. Calculation scheme of subsidence of soil of loess soil with soaking by a descending flow of water under load: 1 - water-saturated soil, 2 - soil of natural moisture, 3- soak front

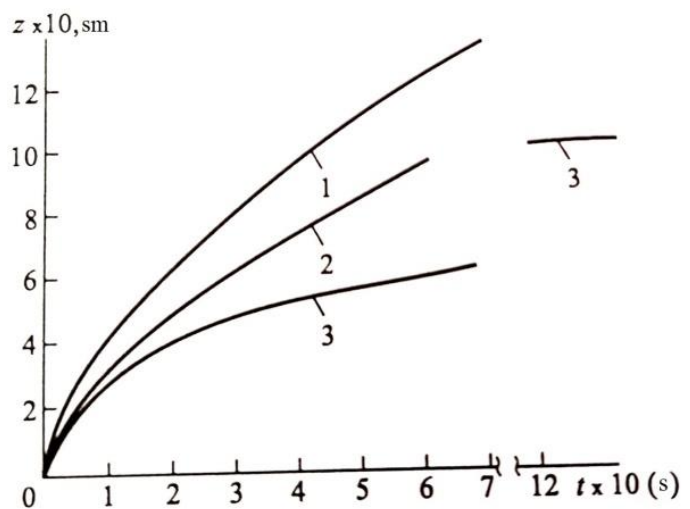

Fig. 2. Curves of infiltration of the downward flow of water without taking into account (1) and taking into account the drawdown (2), and also with the upflow taking into account the drawdown (3). $H^{*}=1 m, k_{f}=10^{-4} \mathrm{sm} / \mathrm{sec}, \gamma=14 \mathrm{kN} / \mathrm{m}^{3}, \gamma_{s}=27 \mathrm{kN} / \mathrm{m}^{3}, \omega_{0} 0,1 ; A_{0}=0,05$, $m_{s l}=0,001 \mathrm{sm}^{2} / \mathrm{N}, \mathrm{p}=20 \mathrm{~N} / \mathrm{sm}^{2}$

In the case of soaking from below, negative friction should be determined from the friction coefficient along the side surface of the piles for $w=w_{0}$, i.e.

where $f_{0}$ - is $f_{w}$ when $w=w_{0}$.

$$
T(t)=U[h-z(t)] f_{0}
$$


Obviously, when soaking from below, the negative friction significantly exceeds friction when soaking from above. Firstly, because $f_{0}>f_{w}$, and secondly, a slight increase in the groundwater level, in order to cause significant friction along the lateral surfaces of the piles. So, if we determine the bearing capacity of piles with negative friction when soaking from below, we get

$$
F_{d}=\gamma_{c}\left(\gamma_{c k} R \cdot A+U \gamma_{c f}\left\{f_{w} z(t)-f_{0}[h-z(t)]\right\}\right)
$$

From this equation it follows that the expression in curly brackets can have a negative value, which reduces the load-carrying capacity of the piles.

\section{One-dimensional compaction of a layer of loess soil with incomplete soaking (moisture transfer)}

In the case of insufficient water, the movement of moisture is not due to a continuous filtration flow of water, but to moisture transfer, which is described in accordance with Fick's law, i.e. the equation of the form:

$$
\frac{\partial w}{\partial t}=c_{w} \nabla^{2} w
$$

where $c_{w}$ is the moisture conductivity coefficient $\left(\mathrm{sm}^{2} / \mathrm{sec}\right), \nabla^{2} w$ is the Laplace operator.

The solution of this problem for the one-dimensional motion of moisture in the soil layer at the initial humidity $w(\mathrm{z})=w_{0}=$ const and under the boundary condition $w(0, t)$ is written as:

$$
w(z, t)=w^{*} \cdot\left[1-\phi\left(\frac{z}{2 \sqrt{c_{w} \cdot t}}\right)\right]
$$

where $\phi(x)$ is the integral exponential function, and $\phi(0)=0, \phi(\infty)=1, \mathrm{w}^{*}=w_{2}-w_{1}$.

And in this case, the progress of moisture has a character that fades in time, since it has a character that decays in time.

In the case where the moisture content on the boundary varies according to the law of the periodicity, i.e. the solution (13) has the form

$$
w(z, t)=w^{*} \cdot \exp \left(-\sqrt{\frac{\omega}{2 \sqrt{c_{w}}}} \cdot z\right) \cdot \cos \left(-\sqrt{\frac{\varpi}{2 \sqrt{c_{w}}}} \cdot z+\omega t\right)
$$

It can be seen that the amplitude of humidity decreases with growth of $z$ according to the exponential law.

In the case of a limited thickness of a layer $\mathrm{h}$ with initial $w(z, t)=w_{0}=$ const, and boundary conditions of the form $w(z, 0)=w_{1}>w_{0}$ and $d w / d z=0$ with $z=h$, we obtain 


$$
w(\mathrm{z}, \mathrm{t})=\mathrm{w} \cdot\left\{1-\frac{4}{\pi} \sum_{i=1}^{\infty} \frac{1}{n} \sin \frac{i \pi z}{2 h} \exp \left(-\frac{\pi^{2} i^{2}}{4 h^{2}} c_{w} t\right)\right\} .
$$

According to the known laws of soil moistening by coordinate and by time $t$, it is possible to determine the SSC of an array of moist loess soil, including the amount of subsidence over time

$$
S_{s l}(t)=z(t) \cdot p(z)\left\{A_{0}[w(z \cdot t)]+m_{s l}[w(z \cdot t)]\right\},
$$

where $-p(z)=p_{0}+\gamma_{s l} \cdot z(t), \quad p_{0}$ - external load, $A_{0}(w), m_{s l}(w)$ - subsidence coefficients for incomplete saturation of pores with water $\left(w<w_{n}\right)$, determined from the results of tests of loess soil at different degrees of saturation of the pores with water.

\section{The interaction of the pile with the subsiding soil}

When the pile interacts with the surrounding subsiding soil, there is a negative friction along its lateral surface, due to which the pile gives an additional draft, and at the lower end of the pile the supporting force increases. To quantify the stress-strain state of the soil surrounding the pile as a calculation model, a two-layer cylinder of a given diameter $d_{c}=2 b$ containing a pile diameter $d_{c}=2 a$. is considered in the present paper. The upper layer of the ground cylinder is subsidence, and the lower one is composed of relatively dense soils. The interaction of an absolutely rigid pile with a sinking soil is considered for the moment when the top layer of the ground cylinder is completely saturated with water, i.e. When the soaking front reached the depth $z=l_{1}$ (Figure 3). At this point, the pile surrounding the pile sags by the amount determined on the basis of formula (10). With depth, it decreases linearly

$$
s_{1}(z)=s_{s}^{\max }\left(1-\frac{z}{l_{1}}\right)
$$

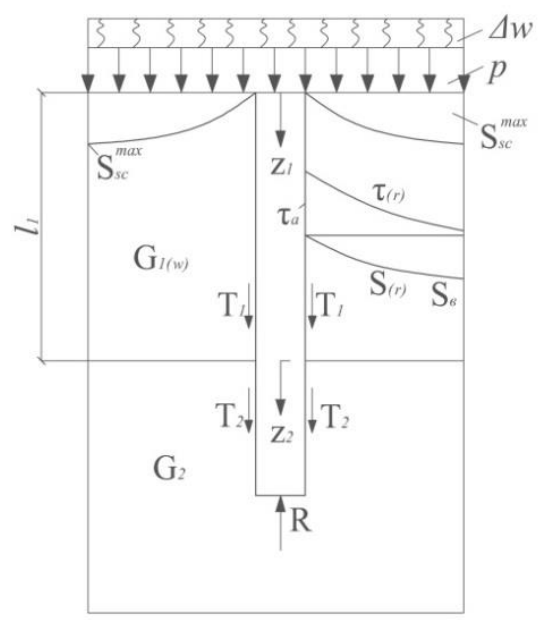

Fig. 3. The design scheme of the pile's interaction with the surrounding subsiding soil 
In the contact area of the pile with the ground, there are angular deformations $\gamma(r, z)$ and tangential stresses $\tau(r, z)$ that are associated with vertical displacement $s(z, r)$ by the dependence

$$
\gamma(z, r)=\frac{d s(z, r)}{d r}=-\frac{\tau(z, r)}{G_{1}(w)}
$$

where $G_{1}(w)$ is the shear modulus, which depends on humidity $w$, and

$$
G_{1}(w)=G_{0}\left(w^{*}-w\right) / w^{*}
$$

It follows from (19) that when $w \rightarrow w^{*} \gamma(z, r) \rightarrow \infty$.

We assume that the resulting tangential stresses $\tau(r, z)$ are taken in the following dependence on $r$

$$
\tau(z, r)=\tau_{a}(z) \frac{(b-r)^{2}}{(b-a)^{2}}
$$

where $\tau_{a}(z)$ is the shear stress of the soil on the lateral surface of the pile.

Substituting (21) into (19) after integration, we obtain

$$
S_{1}(z, r)=\frac{\tau_{n}(z)}{3 G_{1}(w)} \cdot \frac{(b-r)^{3}}{(b-a)^{2}}+C_{1}
$$

where $C_{1}$ is the integration constant, which can be determined by assuming that $S_{2}\left(l_{1}\right)$ is equal to the draft of the circular rigid stamp $S_{c}=S_{s}$, i.e. we have

$$
C_{1}=-\tau_{a}(t) \frac{b-a}{3 G_{1}(w)}+S_{c}
$$

and finally

$$
S_{1}(z, r)=S_{c}-\frac{\tau_{a}(z)}{3 G(w)} \cdot \frac{(b-a)^{3}-(b-r)^{3}}{(b-a)^{2}}
$$

When $r=b$ we get the value of the precipitation on the contour of the cylinder

$$
S_{b}(z)=S_{c}-\frac{\tau_{a}(z)}{3 G_{1}}(b-a)
$$

This deposit is equal to the amount of subsidence outside the ground cylinder (24). Comparing (18) and (25), we find the change in the tangential stress on the pile surface with depth, i.e. we get

$$
\tau_{a}(z)=\left[S_{c}-S_{1}^{\max }\left(1-\frac{z}{l_{1}}\right)\right] \frac{3 G_{1}(w)}{b-a}
$$

The sediment of the underlying non-shrink layer under the influence of the weight of the subsidence layer and the distributed load will be equal to 


$$
S_{2}^{\max }=\left(\gamma_{s e} \cdot l_{1}+p\right) \cdot m \cdot r_{2} \cdot l_{2}
$$

With depth the sediment of the second layer will decrease, i.e.

$$
S_{2}=(z) S_{r}^{\max }\left(1-\frac{z_{2}}{l_{2}}\right)
$$

where $z_{2}$ - the second coordinate in the calculation scheme, introduced for convenience.

The precipitation of the soil around the pile in the second layer can be determined in this way by analogy with the first layer

$$
S_{2}(z, t)=S_{e}-\frac{\tau_{a}\left(z_{2}\right)}{3 G_{2}} \frac{(b-a)^{3}-(l-r)^{3}}{(b-a)^{2}}
$$

when $r=b$ we obtain

$$
S_{2}(z, l)=S_{c}-\frac{\tau_{a}\left(z_{2}\right)}{3 G_{2}}(b-a)
$$

Comparing this value of the draft with the draft (28), we obtain

$$
\tau_{a}\left(z_{2}\right)=\sigma_{c}-S_{2}^{\max }\left(1-\frac{z_{2}}{l_{2}}\right) \cdot \frac{3 G_{2}}{b-a}
$$

To determine the unknowns $S_{a} \tau_{a}(z)$ and $\tau_{a_{1}}\left(z_{2}\right)$ we use the equilibrium condition

$$
T_{1}+T_{2}+\tau \approx R
$$

where

$$
\begin{gathered}
T_{1}=2 \pi a \int_{0}^{l_{1}} \tau_{a}\left(z_{1}\right) d z_{1} ; R=\pi a^{2} \sigma_{R}=\frac{4 a G_{2} S_{c}}{1-v_{2}} \\
T_{2}=\int_{0}^{l_{2}} \tau_{a}\left(z_{2}\right) d z_{2} ; \tau=\pi a^{2} p
\end{gathered}
$$

Substituting the values of $\tau_{a}\left(z_{1}\right)$ and $\tau_{a}\left(z_{2}\right)$ from (26) and (31) into the first two lines of (33) after integration, we obtain

$$
\begin{aligned}
& T_{1}=\frac{\sigma \pi a G_{1} l_{1}}{b-a}\left(S_{c}-S_{s e(1)}^{\max } / 2\right) \\
& T_{2}=\frac{\sigma \pi a G_{2} l_{2}}{b-a}\left(S_{c}-S_{s l(2)}^{\max } / 2\right)
\end{aligned}
$$

Substituting these values of $T_{1}$ and $T_{2}$ in (32) with allowance of $R$ and $p$ for and obtaining finally

where

$$
S_{c}=\frac{\left(1-v_{2}\right)\left(G_{1} l_{1} S_{1}^{\max }+G_{2} l_{2} S_{2}^{\max }\right)+\left(1-v_{2}\right) p / z G_{2}}{G_{1} l_{1}+G_{2} l_{2}-b+a}
$$




$$
\begin{gathered}
S_{1}^{\max }=l_{1}\left[A_{0}+m_{s e}\left(p+\gamma_{s e} l_{1} / 2\right)\right] \\
S_{2}^{\max }=\gamma_{s e} l_{2} m_{2}
\end{gathered}
$$

Substituting these values of $S_{1}^{\max }$ and $S_{2}^{\max }$ in (36), we can determine the values of $\tau_{a}\left(z_{1}\right)$ and $\tau_{a}\left(z_{2}\right)$ by (26) and (31), respectively.

Thus, the task is completely solved. An additional pile precipitation force was determined due to the humidification of the upper layer and the appearance of negative friction. In addition, the timing of the humidification of the upper layer is determined during the filtration flow and moisture transfer.

\section{Conclusion}

Main conclusions:

1. With intensive soaking of loess soils stratum due to technogenic flooding or flooding of territories, subsidence appears that affect the SSC in the loess soil and the underground structures of buildings and structures interacting with it (foundation, pile, etc.)

2. In order to quantify the additional pile settlement over time, the problem of loess base subsidence in the natural state was solved, taking into account the descending and ascending filtration flow or moisture transfer. It is shown that the penetration rate of water in the loess soil is fading.

3. When the pile interacts with the surrounding settling soil along the side surface of the pile, negative friction occurs, which causes additional pile sediment and additional reinforcement at its lower end, which should be taken into account when designing pile foundations in loess soils.

This work was financially supported by the Ministry of Education and Science (state task \#1.4984.2017/6.7). All tests were carried out using research equipment of The Head Regional Shared Research Facilities of the Moscow State University of Civil Engineering.

\section{References}

1. V.T. Trofimov, Soil science (Moscow, Moscow State University, 1024, 2005)

2. M.N. Goldstein, Mechanical soil parameters (Moscow, Stroiizdat, 337, 1973)

3. A.A. Mustafaev, Foundations on subsidence soils (Moscow, Vysshaya shkola, 585, 1989)

4. P.Ya. Poluboyarinova-Kochina, Groundwater movement theory (Moscow, Nauka, 664, 1977)

5. A.N. Tihonov, A.A. Samorskiy, Equations of mathematical physics (Moscow, Nedra, $575,1979)$

6. Z.G. Ter-Martirosyan, Rheological parameters of soils and calculations of construction bases (Moscow, Stroiizdat, 200, 1990)

7. Z.G. Ter-Martirosyan, Soil mechanics (Moscow, ASV, 550, 2009)

8. N.A. Tsytovych, Frozen soils mechanics (Moscow, Vysshaya shkola, 425, 1973).

9. N.A. Tsytovych, Z.G. Ter-Martirosyan, Basics of applied geomechanics in civil engineering (Moscow, Vysshaya shkola, 317, 1981) 